\title{
EndoLISA ${ }^{\circ}$ a novel and reliable method for endotoxin detection
}

\begin{abstract}
A new test for the sensitive detection of endotoxin has been developed, based on a lipopolysaccharideselective, precoated microplate and a factor $\mathrm{C}$-based detection reagent and presented in a complete kit format. The selective capture of lipopolysaccharide (LPS) is achieved using a phage-derived receptor protein exhibiting high affinity and high specificity for the conserved core region of LPS. After binding of sample-LPS to the microplate as the first stage of the assay, the original sample matrix is washed off, thereby eliminating potentially interfering components. In the second stage of the assay, LPS is detected by factor $C$ in a process whereby the principal receptor of the Limulus amoebocyte coagulation cascade reacts with a fluorescence substrate. The new endotoxin test EndoLISA has a detection range from $0.05 \mathrm{EU} / \mathrm{ml}$ up to $500 \mathrm{EU} / \mathrm{ml}$.
\end{abstract}

Endotoxins are heat-stable breakdown products of Gram-negative bacteria. Chemically, endotoxin is LPS, the main constituent of the outer cell membrane ${ }^{1}$. LPS triggers severe physiological reactions, and therefore detection of endotoxin contamination is mandatory in pharmaceutical production and highly relevant in life science and medical research. For years, the Limulus amoebocyte lysate $(\mathrm{LAL})$ assay was the only practical method for direct detection of endotoxin ${ }^{2}$. More recently, a similar assay based on recombinant factor $\mathrm{C}$ has been introduced ${ }^{3}$, avoiding the use of blood from the endangered population of horseshoe crabs. However, both test formats suffer from their status as homogeneous assays that can be materially disturbed (inhibited or stimulated) by many possible constituents of the sample. To overcome this basic limitation, samples must be diluted in order to lower the concentrations of interfering components. For the same reason, spike controls are required in order to prove the validity of a test result. In the face of these drawbacks, the scientific community is constantly seeking improved methods for endotoxin detection ${ }^{4}$.

\section{Solid phase-based test format and its advantages}

ELISA techniques are well established in biochemical analysis and diagnostics. They are sensitive, robust and reliable. Nevertheless, an ELISA-based method for endotoxin detection is not available on the market because no one has succeeded in developing antibodies with

Holger Grallert, Sonja Leopoldseder, Maike Schuett, Peter Kurze \& Bernd Buchberger

Hyglos GmbH, Bernried, Germany. Correspondence should be addressed to H.G. (holger.grallert@hyglos.de). both broad specificity for LPS and sufficient affinity, owing to the fact that LPS is a very heterogeneous substance class.

We have selected a bacteriophage receptor protein targeting the conserved core region of LPS. As in a classical ELISA, the target analyte (LPS) is selectively bound to the surface of the precoated test plate, allowing the sample matrix to be eliminated through a washing step. Subsequently, the LPS is detected by its intrinsic capacity to activate the zymogen form of factor $\mathrm{C}$, which finally generates a fluorescence signal by conversion of a substrate. Using this ELISA-like principle, EndoLISA exhibits clear advantages over the established homogeneous detection methods, including: fewer false-positive results induced by, for example, $\beta$-glucan, proteases or phospholipids, fewer false-negative results caused by inhibitory constituents of the sample,fewer invalid results necessitating re-testing, less interference in complex samples, and therefore higher sensitivity; and broad dynamic range.

\section{Application data and discussion of results}

EndoLISA has a measurement range of four orders of magnitude, from $0.05 \mathrm{EU} / \mathrm{ml}$ to $500 \mathrm{EU} / \mathrm{ml}$ (Fig. 1). This is possible because fluorescence is used as the detection mode and because the sigmoid standard curve can be approximated by a four-parameter curvefitting algorithm. The specified sensitivity limit is $0.05 \mathrm{EU} / \mathrm{ml}$.

It is important that any alternative endotoxin detection method has a good correlation with the established method (the gold-standard LAL assay). Therefore, we compared various types of LPS over a broad concentration range (Fig. 2). The results for different LPS species indicated that EndoLISA has a linear correlation with the LAL assay over the entire measurement range $\left(R^{2}=0.91\right)$. 
Table 1 | Highest tolerated concentrations of substances for valid LPS spike recovery, a comparison between EndoLISA and LAL assay.

\begin{tabular}{|c|c|c|c|c|}
\hline & Substance & Solvent & EndoLISA & LAL assay \\
\hline \multirow[t]{5}{*}{ Buffer/pH } & Acetate $(\mathrm{pH} 4.0)$ & $100 \mathrm{mM} \mathrm{NaCl}$ & $50 \mathrm{mM}$ & $12.5 \mathrm{mM}$ \\
\hline & Acetate $(\mathrm{pH} 5.0)$ & $100 \mathrm{mM} \mathrm{NaCl}$ & $100 \mathrm{mM}^{\mathrm{a}}$ & $12.5 \mathrm{mM}$ \\
\hline & MES (pH 6.0) & $100 \mathrm{mM} \mathrm{NaCl}$ & $100 \mathrm{mM}^{\mathrm{a}}$ & $5 \mathrm{mM}$ \\
\hline & $\begin{array}{l}\text { Potassium phosphate } \\
\qquad(\mathrm{pH} 7.2)\end{array}$ & $100 \mathrm{mM} \mathrm{NaCl}$ & $100 \mathrm{mM}^{\mathrm{a}}$ & $50 \mathrm{mM}$ \\
\hline & Imidazole (pH 7.4) & Water & $500 \mathrm{mM}$ & $40 \mathrm{mM}$ \\
\hline \multirow[t]{2}{*}{ Salt } & $\mathrm{NaCl}$ & Water & $1 \mathrm{M}$ & $0.5 \mathrm{M}$ \\
\hline & $\mathrm{KCl}$ & Water & $1 \mathrm{M}$ & $0.25 \mathrm{M}$ \\
\hline \multirow[t]{2}{*}{ Chaotropic agent } & Urea & Water & $6 M$ & $0.5 \mathrm{M}$ \\
\hline & Guanidinium chloride & Water & $1 \mathrm{M}$ & $0.05 \mathrm{M}$ \\
\hline Organic solvent & Methanol & Water & $20 \%$ a & $5 \%$ \\
\hline \multirow[t]{5}{*}{ Detergent } & SDS & Water & $0.05 \%$ & $0.001 \%$ \\
\hline & CTAB & Water & $0.004 \%$ & $0.0001 \%$ \\
\hline & Zwittergent 3-14 & Water & $0.02 \%$ & $0.005 \%$ \\
\hline & Tween 20 & Water & $2 \%$ & $0.1 \%$ \\
\hline & Triton $\mathrm{X}-100$ & Water & $0.02 \%$ & $0.005 \%$ \\
\hline \multirow[t]{2}{*}{ Chelator } & EDTA (pH 8.0) & Water & $0.4 \mathrm{mM}$ & $0.4 \mathrm{mM}$ \\
\hline & Citrate $(\mathrm{pH} 7.5)$ & Water & $10 \mathrm{mM}$ & $10 \mathrm{mM}$ \\
\hline \multirow[t]{2}{*}{ Protease inhibitor } & Benzamidine & Water & $100 \mathrm{mM}^{\mathrm{a}}$ & $0.1 \mathrm{mM}$ \\
\hline & PMSF & 2-Propanol & $5 \mathrm{mM}$ & $<0.05 \mathrm{mM}$ \\
\hline Antibiotic & Rifampicin & Methanol & $3.5 \mathrm{mg} / \mathrm{ml}$ & $0.04 \mathrm{mg} / \mathrm{ml}$ \\
\hline
\end{tabular}

aHighest concentration tested.

The tested substances were dissolved in the respective solvents, and dilution series of the samples were prepared in water or $0.1 \mathrm{M}$ NaCl and subsequently spiked with 5 EU/mI LPS (Escherichia coli O55:B5). $\mathrm{EU} / \mathrm{ml}$ values and spike recovery (\%) were calculated. The validity criterion of spike recovery was 50-200\%. CTAB, cetyl trimethylammonium bromide; DMSO, dimethylsulfoxide; MES, methyl ethyl sulfonate; PMSF, phenylmethylsulfonylfluoride; SDS, sodium dodecyl sulphate.

To prove the robustness of EndoLISA, we tested various substance classes commonly used in biological buffer systems (Table 1). The results show that, compared to the LAL assay, EndoLISA is more permissive of high-salt conditions and also tolerates higher concentrations of chaotropic agents and organic solvents. Even in the presence of $6 \mathrm{M}$ urea, the validity criterion was achieved. EndoLISA is functional in a $\mathrm{pH}$ range between $\mathrm{pH} 4$ and $\mathrm{pH} 9$ and at buffer concentrations of up to $100 \mathrm{mM}$. Moreover, detergents are known to affect the micelle structure of LPS and in doing so to modulate the activation of factor $\mathrm{C}^{5}$; very low concentrations of detergent enhance this activation, whereas higher concentrations cause inhibition. As shown, however, EndoLISA tolerates between 4 and 50 times higher detergent concentrations than the LAL assay.

Bivalent cations are essential for the functionality of both the LAL assay and EndoLISA. Therefore, chelating agents such as EDTA or citrate must be diluted below a certain concentration level or, in EndoLISA, can simply be neutralized by the addition of magnesium.

The above results indicate that in the EndoLISA test the negative influence of sample constituents is substantially smaller than for the LAL assay. Because of its solid phase-based test format, EndoLISA is more robust and can be applied to complex sample matrices without extensive dilution.

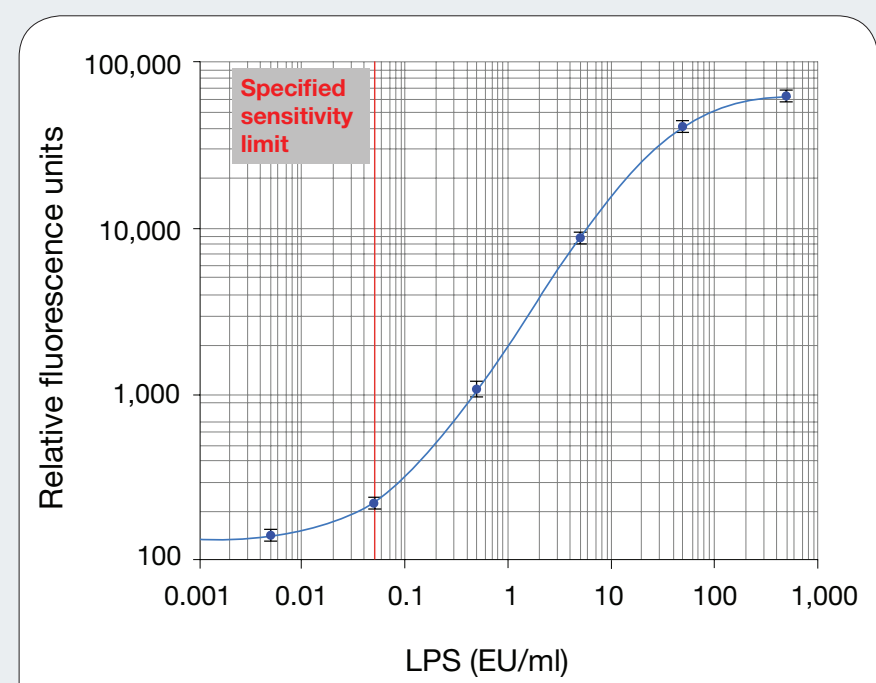

Figure 1 | Standard curve of the EndoLISA test: concentrations of the LPS standard are plotted against the relative fluorescence signal. EU, endotoxin unit. 


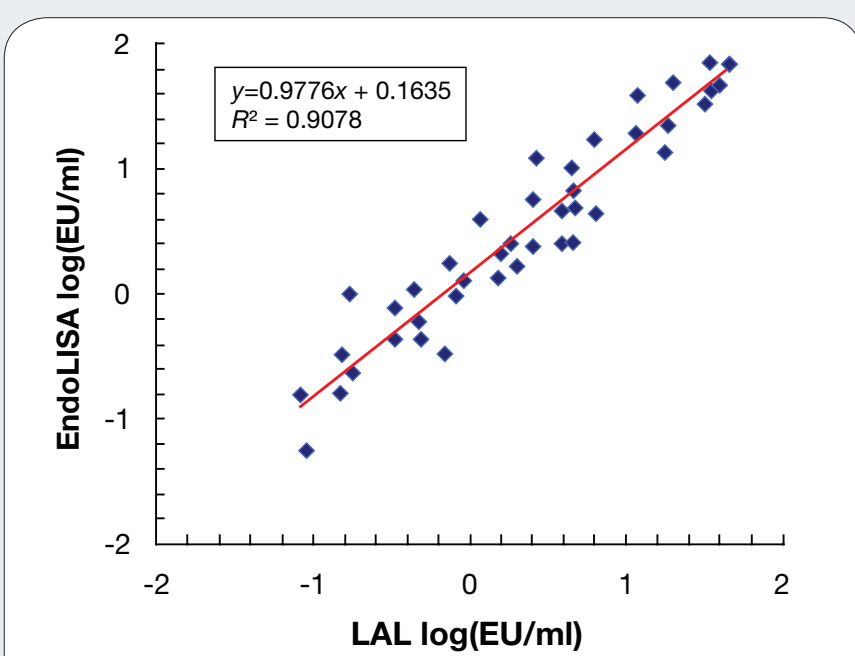

Figure $\mathbf{2}$ | Correlation plot of EndoLISA versus LAL assay. Multiple LPS preparations and dilutions from different sources, including LPS of mutant strains, were compared. The tested LPS samples were E. coli O55:B5, E. coli O111:B4 (phenol extract and EDTA extract), E. coli O128:B12, E. coli O45, E. coli K235, E. coli EH 100 (Ra mutant), E. coli J5 (Rc mutant), E. coli F583 (Re mutant), Salmonella enterica serotype Minnesota (wild type and Re mutant), S. enterica serotype Enteritidis, S. enterica serotype Abortus equi, S. enterica serotype Typhimurium, Klebsiella pneumoniae, Serratia marcescens and Pseudomonas aeruginosa serotype 10. Individual dilutions of the LPS samples were prepared in water. The coefficient of correlation $\left(R^{2}\right)$ was determined by linear regression.

\section{Perspectives}

At present there are some areas in which endotoxin detection is not possible or requires cumbersome sample preparation methods. One such area is the detection of endotoxin in human body fluids, such as blood and serum or plasma ${ }^{6}$. One of our short-term goals is to evaluate EndoLISA for direct measurement in such clinical samples and also to provide protocols for diagnostic applications.

\section{ACKNOWLEDGMENT}

We thank the German Federal Ministry of Economics and Technology, which funded the development of EndoLISA (ZIM Project).

1. Brade, H. et al. (eds). Endotoxin in Health and Disease (Marcel Dekker, New York, 1999).

2. Pfeiffer, M. \& Weiss, A.R. Removal of LAL_-Test interfering low molecular weight substances by ultrafiltration. in Detection of Bacterial Endotoxins with the Limulus Amoebocyte Lysate Test (ed. Novitsky, T.J.) 251-262 (Alan R. Liss, New York, 1987).

3. Loverock, B. et al. A recombinant factor $C$ procedure for the detection of Gramnegative bacterial endotoxin. Pharmacopeial Forum 36, 321-329 (2010).

4. Sakata, M., Fukuma, Y., Todokoro, M. \& Kunitake, M. Selective assay for endotoxin using poly(epsilon-lysine)-immobilized Cellufine and Limulus amoebocyte lysate (LAL). Anal. Biochem. 385, 368-370 (2009).

5. Nakamura, T., Tokunaga, F., Morita, T. \& Iwanaga, S. Interaction between lipopolysaccharide and intracellular serine protease zymogen, factor C, from horseshoe crab (Tachypleus tridentatus) hemocytes. J. Biochem. 103, 370-374 (1988).

6. Hurley, J.C. Endotoxemia: methods of detection and clinical correlates. Clin. Microbiol. Rev. 8, 268-292 (1995). 